\title{
Healthcare system barriers of breast and thyroid screening for childhood and young adulthood cancer survivors in France: A qualitative study of the DeNaCaPST programme at one year
}

Demoor-Goldschmidt $\mathrm{C}^{1,2 *}$, Berger $\mathrm{C}^{3}$, Guichard $\mathrm{I}^{4}$, Supiot $\mathrm{S}^{5}$, Dezellus $\mathrm{A}^{6}$, Filhon $\mathrm{B}^{7}$, Bondiau ${ }^{8}$, M Poirée PY, Oudot $\mathrm{C}^{10}$, Claude $\mathrm{L}^{11}$, Truc $\mathrm{G}^{12}$, Briandet $\mathrm{C}^{13}$, Padovani $\mathrm{L}^{14}$, Coze $\mathrm{C}^{15,16}$, Bernier $\mathrm{V}^{17}$, Kerr $\mathrm{C}^{18}$, Reguerre $\mathrm{Y}^{19}$, Sudour-Bonnange $\mathrm{H}^{20}$, Gaudichon $\mathrm{J}^{21}$, Vigneron $\mathrm{C}^{22}$, Dumas $\mathrm{A}^{23}$ and de Vathaire $\mathrm{F}^{1}$

${ }^{1}$ Centre for Research in Epidemiology and Population Health (CESP), Cancer and Radiation team, Institut nationale de la recherche médicale (INSERM) U1018, Université Paris-Sud, UVSQ, Université Paris-Saclay, 94807 Villejuif, France

${ }^{2}$ Department of Pediatric Hematology and Oncology, Angers University Hospital, Angers, France

${ }^{3}$ Department of Pediatric Hematology and Oncology, Nord saint Etienne University Hospital, cedex, 42055 St Etienne, France

${ }^{4}$ Department of Internal Medicine Unit, University Hospital, Saint-Etienne, France

${ }^{5}$ Department of Radiation Oncology, Institut de Cancérologie de l'Ouest, Blvd J. Monod, 44805 Saint Herblain, France

${ }^{6}$ Department of oncology, Institut de Cancérologie de l'Ouest, Bd Jacques Monod, 44805, Saint Herblain, France

${ }^{7}$ Department of Pediatric Hematology and Oncology, Rouen University Hospital, Rouen, France

${ }^{8}$ Department of Radiation Oncology, Centre Antoine Lacassagne, Nice, France

${ }^{9}$ Department of Pediatric Hematology and Oncology, University Hospital L'Archet, Nice, France

${ }^{10}$ Department of Pediatric Hematology and Oncology, University Hospital Limoges, Limoges, France

${ }^{11}$ Department of Radiation Oncology, Centre Léon Bérard, Lyon, France

${ }^{12}$ Department of Radiation Oncology, Paul Strauss Center, Strasbourg, France

${ }^{13}$ Department of Pediatrics, University Hospital of Dijon, Dijon, France

${ }^{14}$ Department of Radiation Oncology, Hôpital d'enfants La Timone, Marseille, France

${ }^{15}$ Department of Pediatric Hematology and Oncology, Assistance Publique Hôpitaux de Marseille (APHM), Hôpital d'Enfants de La Timone, Marseille, France

${ }^{16}$ Aix-Marseille Université, France

${ }^{17}$ Department of Radiation Oncology, Institut de Cancérologie de Lorraine, Nancy, France

${ }^{18}$ Department of Radiation Oncology, institut du cancer de Montpellier, Montpellier, France

${ }^{19}$ Department of Pediatric Hematology and Oncology, CHU de Saint Denis de La Réunion, Saint Denis, France.

${ }^{20}$ Pediatric Oncology Unit, Children, Adolescents and Young Adults Unit, Oscar Lambret Center, Lille, France

${ }^{21}$ Department of Pediatric Hematology and Oncology, CHU Caen, Caen, France.

${ }^{22}$ Department of Radiation Oncology, Centre Paul Strauss, Strasbourg, France

${ }^{23} \mathrm{ECEVE}$, Institut nationale de la recherche médicale (Inserm) UMRS 1123, université Paris-Diderot, Paris, France

\begin{abstract}
Purpose: Childhood, adolescent and young adult cancer survivors (CAYACS) who were treated by radiotherapy have a significant risk of developing subsequent malignancies, particularly breast and thyroid cancers when the field of irradiation concerned these organs. Advice regarding their increased risk of developing secondary breast (SBC) and thyroid (STC) cancer are recommended to ensure risk-stratified life-long follow-up care including appropriate screening. In France, a national program called $\mathrm{DeNaCaPST}$ was started to promote this care. We aimed to explore the perspectives of medical practitioners (MP) on the healthcare system factors that limited inclusion in this programme.

Methods: Data of the DeNaCAPST programme regarding organisation were studied, including data from a qualitative survey done among MP.

Results: Seventeen months after the DeNaCaPST programme started, $84.6 \%$ of the French regions had participating centres/hospitals, along with one overseas territory. The main barriers to screening highlighted by MP were: 1 ) inconvenient and under-resourced healthcare professionals to perform these consultations, (2) difficulty determining which CAYACS need SBC and/or STC screening, (3) difficulty organising the network of professionals from paediatric to adult health services.

Conclusions: Despite a slow inclusion speed and a suboptimal geographical coverage, changes are underway and should allow for several improvements and interesting perspectives for this study. CAYACS lost to follow-up are difficult to invite for a consultation. The absence of real long-term follow-up care is a real barrier to promoting such screening, which must involve a transition care between the paediatric oncologists who know who are at risk and why and the adult medical professionals who have the knowledge to do the screening. The involvement and empowerment of CAYACS is necessary to promote patient-centred healthcare solutions and seems feasible.
\end{abstract}

${ }^{\star}$ Correspondence to: Charlotte demoor-goldschmidt, Centre for Research in Epidemiology and Population Health (CESP), Cancer and Radiation team, Institut nationale de la recherche médicale (INSERM) U1018, Université Paris-Sud, UVSQ, Université Paris-Saclay, 94807 Villejuif, France, E-mail: charlotte.demoor@inserm.fr

Key words: survivorship, childhood cancer survivor, oncology, radiotherapy, breast cancer screening, thyroid cancer screening, qualitative. health care system

Received: March 09, 2020; Accepted: March 30, 2020; Published: April 02, 2020 
Demoor-Goldschmidt C (2020) Healthcare system barriers of breast and thyroid screening for childhood and young adulthood cancer survivors in France: A qualitative study of the DeNaCaPST programme at one year

\begin{abstract}
Abbreviations: ACS: American Cancer Society; ARC: Association for Research against Cancer; CAYACS: Childhood; adolescent and young adult cancer survivors; CCTIRS: Advisory Committee on the Treatment of Research Information in the Field of Health; CNIL: French control authority for the protection of personal data; COG: Children's Oncology Group; ERR: Excess of Relative Risk; FCCSS: French Childhood Cancer Survivor Study; FNA: Fine-Needle Aspiration; GFRP: French group of paediatric radiation oncologists; Gy: Gray; LTFU: Long Term Follow Up; MP: Medical practitioners; MRI: Magnetic resonance imaging; NCI: National Cancer Institute; SBC: Secondary breast cancer; SFCE: French Society of Childhood Cancers; STC: secondary thyroid cancer; UK CCSG: Children's Cancer and Leukaemia Group
\end{abstract}

\section{Introduction}

Radiation therapy during childhood or young adulthood is an established risk factor for secondary breast (SBC) or thyroid (STC) cancer [1-3]. The cumulative risk for a breast cancer after mediastinal irradiation above $20 \mathrm{~Gy}$ is similar to that for BRCA2-mutated women: around $35 \%$ at 40 years [4]. Breast cancer screening for childhood, adolescent and young adult cancer survivors (CAYACS) is the subject of numerous articles. Many institutions, including the COG (Children's Oncology Group), UK CCSG (Children's Cancer and Leukaemia Group), NCI (National Cancer Institute), ACS (American Cancer Society), HAS (French High Authority of Health) and SFCE (French Society of Childhood Cancer) recommend breast screening for women at a high and early risk; this was resumed in the harmonisation of international guidelines [1,5]. Hodgson, et al. developed a mathematical model to evaluate the benefit of early-initiated breast screening on SBC mortality starting at 25 years of age compared with screening initiated at 40 years of age, which would be even later in France without any organised program (national breast screening starts at the age of 50) [6]. Early MRI-based screening should reduce SBC mortality at the age of 75 from $16.65 \%$ with no early screening to $15.38 \%$ in the case of same-day annual mammography and MRI (16.28\% with annual mammography, $15.40 \%$ annual MRI), leading to the prevention of one SBC death for every approximately 80 patients screened. Due to an excellent cure rate for breast cancer, the difference, even if it is significant, can be insufficient. In comparison, studies on the general population suggest that 1339 women aged between $50-59$ years need to be invited to a screening program to prevent 1 breast cancer death [7]. The UK Breast screening program prevents 5.7 breast cancer deaths for every 1000 women screened in a period of 20 years. The Norwegian program has calculated 5.7 prevented deaths in a period of 29 years, while this model suggests that 13.92 breast cancer deaths are prevented in a 24-year period of screening [8,9]. In the case of SBC diagnosed from 25 to 39 years, same-day annual mammography in addition with MRI was found to reduce SBC mortality at the age of 75 years to $59.09 \%$ versus $71.38 \%$ without early screening. This model assumed that SBC arises with a similar growth rate as cancers in the general population and are not more aggressive. It also assumed that $65 \%$ of women were fully compliant with the screening, $25 \%$ attended half of their annual screenings and $10 \%$ were non-compliant. Nevertheless, more than mortality, we can expect an improvement regarding morbidity and quality of life because smaller cancers are diagnosed before the age of $40:<2 \mathrm{~cm}$ in $34.12 \%$ without early screening versus $81.66 \%$ with sameday annual mammography and MRI; diagnosed with positive nodes: $44.15 \%$ without any early screening versus $11.73 \%$ with same-day annual mammography and MRI. This is in line with our previous study [10]. Regarding thyroid cancer screening, the international group for harmonisation of guidelines recommends that CAYACS should be counselled regarding their increased risk of STC and their options for STC surveillance [3]. The decision to start screening should be made by the health care provider in consultation with the CAYACS after careful consideration of the survivor's perspective about the advantages and disadvantages of STC screening, which can be either done by neck palpation or by ultrasonography.

The optimal healthcare services model for long-term follow-up care (LTFU) for CAYACS is still debated [11,12]. In France, various models of care have been established: in some cities, they access LTFU care with adult healthcare professionals, while in others it depends of the paediatric oncology department or radiotherapy department $[12,13]$. In most cities, identified LTFU consultations are not organised for CAYACS after a solid cancer and, these patients are followed by their general practitioner who is likely to be unfamiliar with survivor followup recommendations. Barriers to LTFU dedicated care are influenced by the financing, regulation, and provision of health actions/services.

Understanding the healthcare system factors that slow down the number of CAYACS inclusions is necessary to helps to develop tools to ameliorate practice regarding patient-centred needs and expectations, with the aim of delivering accessible, acceptable, appropriate and effective cancer screening. Thus, our aim in this study was to examine the views of involved medical practitioners (MP) in the funded DeNaCaPST programme regarding healthcare system factors that complicate breast and thyroid cancer screening and to present and discuss the evolution of the programme.

\section{Methods}

DeNaCaPST is a non-interventional study testing the feasibility of a national screening for thyroid cancer and breast cancer in CAYACS regarding the follow-up and compliance with international recommendations (ClinicalTrials.gov Identifier: NCT03183401). This programme is coordinated by the INSERM 1018 unit in cooperation with the long term follow-up committee of the SFCE and the French group of paediatric radiation oncologists (GFRP). It is funded by the ARC foundation (Association for Research against Cancer).

The final protocol and its amendments have been approved by the CNIL (French control authority for the protection of personal data), the CCTIRS (Advisory Committee on the Treatment of Research Information in the Field of Health) and the ethics committee of INSERM. Included CAYACS received information and oral and written consent was obtained before inclusion in the study.

The general organisation of the programme has already been published [5].

\section{Qualitative data on organisation (MP)}

Data concerning organisation have been collected from the dedicated software. Moreover, an online questionnaire has been sent to the different participating centres; one answer per centre was collected between 12 and 18 months after the programme started. Prior to these MP questionnaires, interviews were conducted with all participant MP. This led to amendments to the programme.

A descriptive analysis was performed using the statistical software XLSTAT.

\section{Results}

Seventeen months after the programme started, it included 20 centres allocated in 19 cities, including one overseas territory covering 11 out of 13 regions (no centre in Corse or in Région Centre) (Figure 1). At the 


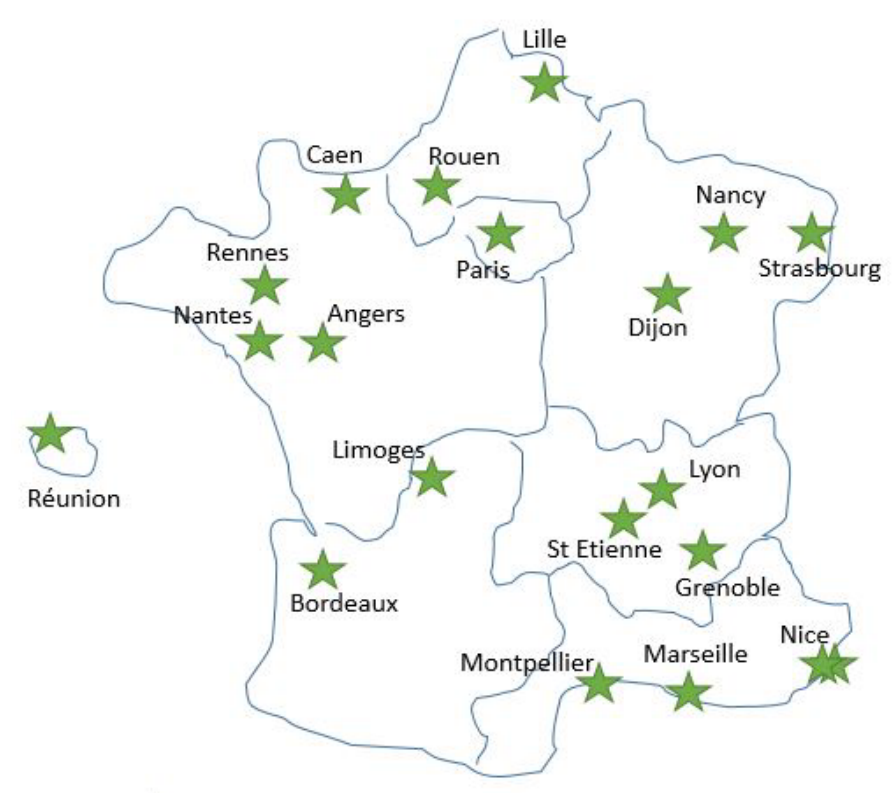

Including centers

Figure 1. Centres participating in the DeNaCaPST programme in April 2019, 17 months after its start

time of this analysis, two centres had opened 12 months ago or more, and four between 4 and 0 months ago; all the opened centres/hospitals are SFCE centres and have prioritised the inclusion of childhood cancer survivors. Ten centres $(52.6 \%)$ have included one patient or more. However, one city has decided not to continue because of insufficient help/tools to perform the study correctly, and which needs more time to find and invite the CAYACS to this screening.

In $73.7 \%(14 / 19)$, the inclusion of patients is organised by paediatric oncologists, in $42.1 \%$ (8/19) by radiotherapy oncologists and in $21.1 \%(4 / 19)$ by adult care doctors (gynaecologists, endocrinologists or internal practitioners).

Questionnaires regarding organisation have been completed by 15 cities out of 18 invited (two were not solicited as the formal start of their participation was between 0 and 2 weeks before the collection of the data). The main barrier to inclusion raised in this survey was the difficulty in listing people at risk: on a scale from 1 (not difficult) to 5 (very difficult), $87.5 \%$ of the centres gave a score from 3 to 5 . Actually, and for the moment, in $33.3 \%$ of centres, the MP have planned to only propose the DeNaCaPST programme to patients coming in for consultations, without searching for previous patients at risk of being lost to follow-up. The other main barriers raised by half of the centres were the difficulty in determining the vital status of the patients listed as at risk before inviting them to an LTFU consultation, finding a new address when the patient has moved and the best way to organise follow-up after a first dedicated LTFU consultation. In $44.9 \%$, in centres who have invited former patients lost to follow-up, the MD stated that an eventual refusal of CAYACS to come to such a consultation after having been solicited is not a major problem, and in $66.7 \%$ of centres, the MD did not experience patient refusals to participate in the DeNaCaPST programme (22.2\% rarely the case, and $11.1 \%$ half the time); all MD declared that all their CAYACS accepted the screening. Regarding each screening, in $87.5 \%$, thyroid cancer screening was not a barrier as it is simple to prescribe and to find radiologists able to do it. For breast cancer screening, in $35.7 \%$ of centres, the network to organise it in this specific population of CAYACS already existed and was formally created for this programme in the other cities; also, $50 \%$ of the interviewed centres stated that breast cancer screening was not a problem in terms of including CAYACS, whereas it was a moderate or high barrier to participation in $12.5 \%$ and $37.5 \%$ of centres, respectively.

According to the MD, participating in a study such as the DeNaCaPST programme which involved completing forms and questionnaires is not a major barrier, even if $50 \%$ of the cities declared that it takes moderately to too much time (score 3 to 5 on a scale of 5), in contrast to finding at-risk patients, inviting them, seeing them in consultations and organising the follow-up. The main missing data needed to screen at-risk patients or to filling the data required for the study was the estimation of the received dose of irradiation on the thyroid or on the mammary buds or breasts.

Concerning the prospective follow-up and on-going screening, $71.4 \%$ of the cities declared they will continue to see their CAYACS and for the others, another MD (adult care professionals) will be involved (50\% declared the involvement of a hospital MD and 50\% declared the involvement of a general practitioner); $80 \%$ of the interviewed MD agreed that there is not a formal way to organise this, but it really depends on the patient.

For the ten centres that had not started to include patients, six answered the questionnaire. According to all of them, the main barrier was a lack of human time to do this screening, and that it was not directly connected to a financial problem. For $66.7 \%$ of these centres, the other main highlighted barrier was the actual absence of a network of involved professionals to do the screening.

For the DeNaCaPST programme, medical software was developed with the aim of helping professionals and to allow for the sharing of medical information between several doctors. Actually, the main user is the MD who includes the patient (Figure 2). When taking into account just centres who have already included patients, they all found it useful, i.e. very to moderately useful to create a summary of the history of cancer in their patients, in $66.7 \%$ and $33.3 \%$ of centres, respectively, and to create a personalised long-term follow-up plan in $50 \%$ and $50 \%$ of centres, respectively. Perspectives concerning this software developed with a group of $\mathrm{MD}$, researchers and patient associations include personal reminders to the MD and the CAYACS and dedicated access for the CAYACS to their medical information; $57.1 \%$ of the 14 MD who participated to this survey found that this is an important and useful perspective, but $28.6 \%$ answered that they do not have the time to use this tool. The remainder had no specific opinion.

\section{Discussion}

MP considered that cancer screening is feasible and acceptable even if it is a long consultation, that rekindles fears. Three themes highlighted MP perspectives of healthcare system factors that functioned as barriers to offering national breast and thyroid screening: (1) inconvenient and under-resourced healthcare professionals to take on these consultations, (2) the difficulty of finding which CAYACS need SBC and/or STC screening and (3) the difficulty in organising a network of professionals from paediatric to adult health services. Another theme can be highlighted, which is the insufficient communication about late effects and cancer screening. On one hand, communication between professionals 17 months after opening, as only patients treated for paediatric cancer were included and no adult oncology or haematology services are currently included; on the other hand, communication between the MP and CAYACS as $25.0 \%$ and $8.4 \%$ of 
Demoor-Goldschmidt C (2020) Healthcare system barriers of breast and thyroid screening for childhood and young adulthood cancer survivors in France: A qualitative study of the DeNaCaPST programme at one year

CAYACS, respectively, included in the DeNaCaPST programme think that they are at equal or decreased risk of SBC/STC. As the network of specialists is becoming more organised, a focus on increasing the screening participation rate of the target population is the next step to maximise the reduction in mortality/morbidity. This implies improved communication and service delivery strategies, including improving the understanding of CAYACS regarding SBC/STC screening with its benefits and potential harms.

The lack of time for MP to identify patients at risk and to provide comprehensive quality care is also an issue. One other problem raised is in regards to finding these children who have become adults and can have moved or changed their second name, which increase the difficulty of inviting CAYACS to these consultations. To address these difficulties, some centres $(1 / 3)$ opted initially for a prospective inclusion, on a case-by-case basis and informed by consultations. To reach the CAYACS concerned, different strategies of communication have been developed, including oral communication in popular scientific conferences and communication via associations (websites, social networks). Professional communications (articles, information via medical news journals, newsletter, mail and presentations at conferences) have also been established to raise awareness among professionals of the risk of secondary cancer, particularly breast and thyroid cancer among CAYACS, and to draw their attention to the fact that screening is recommended and can be done as part of a national DeNaCaPST study. An email address has been created to answer and possibly direct CAYACS to a participating centre (denacapst@inserm. fr). To help CAYACS, an amendment has been proposed and accepted 13 months after its beginning, which allows any physician (private or hospital) to directly request to include a patient if he/she is at risk. Four months later, one private doctor has participated. Also, with the same aim of finding the CAYACS concerned, work was carried out with the French database Pedia-RT, which is a database that prospectively records all radiotherapy performed in children since 2012, so that an automatic email is generated annually to paediatric radiotherapists listed in this database to find the patients eligible for DeNaCaPST programme [12]. Another link will be made with the FCCSS (French childhood cancer survivors study) cohort; this was planned in the initial protocol, but it was not effective during the first year of implementation of the programme due to administrative delays.

Another major difficulty is in estimating the received dose on the organs at risk in former patients. Because of this, a consensus has been proposed by the GFRP to help promote this screening. An amendment has been made to allow the inclusion of CAYACS without precise dosimetric data if they reach a clinical inclusion criteria based on typical radiation treatments, depending on the dose, field and age of the patient (Table 1).

To help to organise the network, several communications have been made, especially to radiologists. The developed software can also be used by them to know more about their patients and to provide results directly. Work is ongoing between professionals and patient associations to develop a user-centred patient interface for the software, which would provide significant value to CAYACS and make them more autonomous and responsible for their health. Many CAYACS are interested in having access to their own medical records [14]. Oeffinger, et al. showed in a study centred on childhood Hodgkin lymphoma survivors that a mailed one-page survivor care plan increases the self-

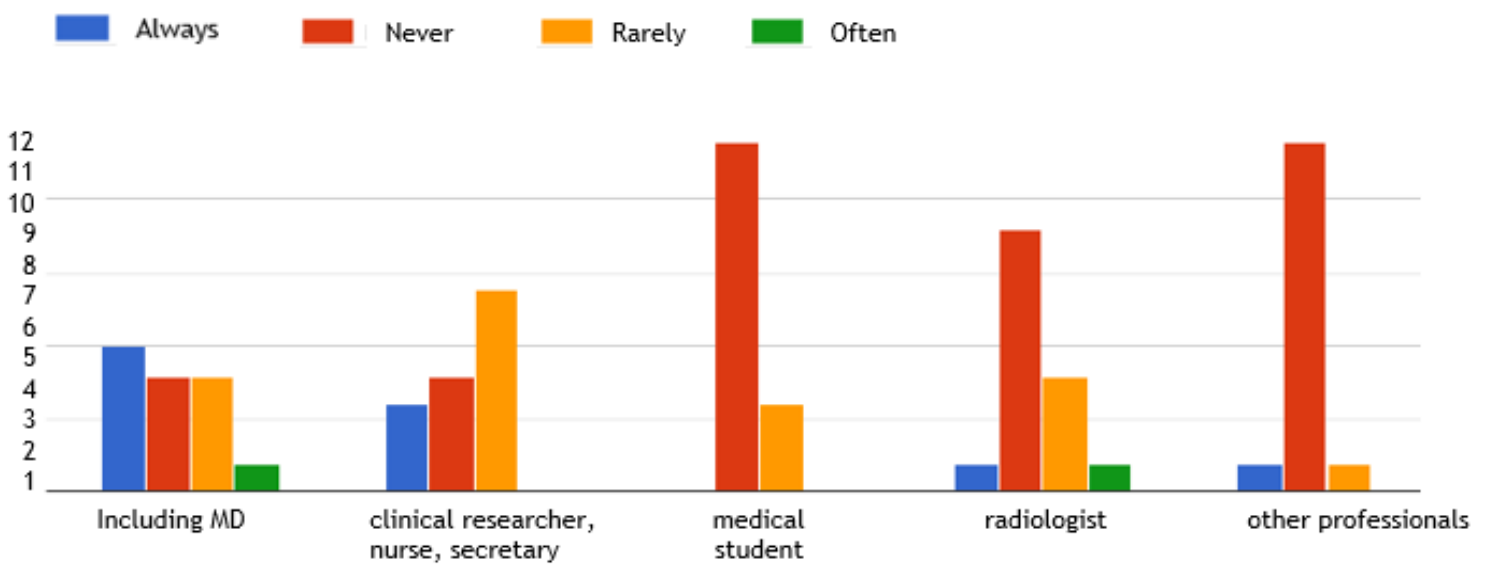

Figure 2. Use of the medical software

Table 1. Inclusion criteria without a specific dose estimate on the breast or thyroid

\begin{tabular}{|c|c|c|}
\hline $\begin{array}{l}\text { Screening possible even if precise dosimetric data are not } \\
\text { found/possible }\end{array}$ & SBC screening & STC screening \\
\hline Irradiation of posterior fossa $\geq 40 \mathrm{~Gy}$ & No & $\begin{array}{l}\text { Yes, unless there is disagreement with local radiotherapy } \\
\text { oncologists }\end{array}$ \\
\hline $\begin{array}{l}\text { Mantle irradiation } \geq 30 \text { Gy } \\
\text { With or without subdiaphragmatic irradiation }\end{array}$ & Yes & $\begin{array}{l}\text { Yes, if at least one cervical or supra-clavicular area is } \\
\text { included }\end{array}$ \\
\hline $\begin{array}{l}\text { Mantle irradiation [20-29] Gy } \\
\text { With or without subdiaphragmatic irradiation }\end{array}$ & Yes, if the girl was pubescent during radiotherapy & $\begin{array}{l}\text { Yes, if at least one cervical or supra-clavicular area is } \\
\text { included }\end{array}$ \\
\hline Cranio-spinal irradiation: mobile junction, photons & $\begin{array}{l}\text { Yes, if the girl was pubescent during radiotherapy } \\
\text { Otherwise, local radiotherapy advice is required }\end{array}$ & ( \\
\hline Prophylactic cerebral field up to the second cervical vertebra & Yes & $\begin{array}{l}\text { No, if radiotherapy was delivered over the age of } 4 \text { years old; } \\
\text { below that, seek local radiotherapist advice. }\end{array}$ \\
\hline Thymus, rib or lung irradiation & Yes & Estimation is required \\
\hline
\end{tabular}


Demoor-Goldschmidt C (2020) Healthcare system barriers of breast and thyroid screening for childhood and young adulthood cancer survivors in France: A qualitative study of the DeNaCaPST programme at one year

reported rates of mammogram and echocardiogram screening [15]. A web-based summary and personalised LTFU care plan accessible by different MP and CAYACS has already been tested and can serve as an effective communication vehicle to provide adequate shared care by different MP, including adult and paediatric MP and may be useful to CAYACS to promote adhesion to LTFU care [16]. In a recent integrative review, educational services, including electronic survivorship care plan services and electronic patient-reported outcome services, were two main components of empowerment among CAYACS $[17,18]$. Receipt of a comprehensive care summary and follow-up plan is the basis to promote appropriate risk-based follow up care; every MP agrees that this is facilitated for patients included in the DeNaCaPST programme [13,19-24].

In conclusion, although the inclusion speed is slow and geographical coverage remains suboptimal, change is underway and should allow for several improvements and interesting perspectives for this study, in particular concerning the study of concerned patients and the organisation of follow-up by highlighting the importance of empowerment of the patient to manage his or her health.

\section{Declarations}

Ethics approval and consent to participate: The final protocol has been approved by the CNIL (French control authority for the protection of personal data), the CCTIRS (Advisory Committee on the Treatment of Research Information in the Field of Health) and ethics committee of the Inserm [16-29]. Informed consent are obtained from each patient in oral and written form before inclusion.

\section{Consent for publication}

Not applicable.

\section{Availability of data and material}

The datasets used and/or analysed during the current study are available from the corresponding author on reasonable request.

\section{Competing interests}

The authors declare that they have no competing interests.

\section{Funding}

The study was funded by the foundation ARC (Association for Research against Cancer). They evaluated the study but were not involved in the design of the study, nor in writing the manuscript.

\section{Authors' contributions}

CDG, FDV, AD, CB, VB, PYB, BF, CK, CO, SP, HSB, CV were involved in designing the DENACAPST study. CDG, FDV, AD were involved in designing the questionnaire. $\mathrm{CDG}$ analysed the results of the questionnaire. CDG, $\mathrm{AD}$ and FDV were involved in drafting the manuscript. All authors have read and approved the manuscript and all except FDV and AD are concerned MP.

\section{Acknowledgements}

We thank the ARC foundation, the SFCE, and Dalhia Khnafo \& Nadia Khaji from Epiconcept, Angela Jackson for the software, the INCa-ARC-Ligue_11902 for their fundings which will permit the further development.

\section{References}

1. Mulder RL, Kremer LC, Hudson MM, Bhatia S, Landier W, et al. (2013) Recommendations for breast cancer surveillance for female survivors of childhood, adolescent, and young adult cancer given chest radiation: a report from the International Late Effects of Childhood Cancer Guideline Harmonization Group. The Lancet Oncology 14: e621-629.

2. Kremer LC, Mulder RL, Oeffinger KC, Bhatia S, Landier W, et al. (2013) A worldwide collaboration to harmonize guidelines for the long-term follow-up of childhood and young adult cancer survivors: a report from the International Late Effects of Childhood Cancer Guideline Harmonization Group. Pediatr Blood Cancer 60: 543-549.

3. Clement SC, Kremer LCM, Verburg FA, Simmons JH, Goldfarb M, et al. (2018) Balancing the benefits and harms of thyroid cancer surveillance in survivors of Childhood, adolescent and young adult cancer: Recommendations from the international Late Effects of Childhood Cancer Guideline Harmonization Group in collaboration with the PanCareSurFup Consortium. Cancer Treat Rev 63: 28-39.

4. Moskowitz CS, Chou JF, Wolden SL, Bernstein JL, Malhotra J, et al. (2014) Breast cancer after chest radiation therapy for childhood cancer. J Clin Oncol 32: 2217-2223.

5. Demoor-Goldschmidt C, Drui D, Doutriaux I, Michel G, Auquier P, et al. (2017) A French national breast and thyroid cancer screening programme for survivors of childhood, adolescent and young adult (CAYA) cancers - DeNaCaPST programme. BMC Cancer 17: 326

6. Hodgson DC, Cotton C, Crystal P, Nathan PC (2016) Impact of Early Breast Cancer Screening on Mortality Among Young Survivors of Childhood Hodgkin's Lymphoma. J Natl Cancer Inst 108: 1

7. Hendrick RE, Helvie MA (2012) Mammography screening: a new estimate of number needed to screen to prevent one breast cancer death. AJR Am J Roentgenol 198: 723-728.

8. Duffy SW, Tabar L, Olsen AH, Vitak B, Allgood PC, et al. (2010) Absolute numbers of lives saved and overdiagnosis in breast cancer screening, from a randomized trial and from the Breast Screening Programme in England. J Med Screen 17: 25-30.

9. Hofvind S, Roman M, Sebuodegard S, Falk RS (2016) Balancing the benefits and detriments among women targeted by the Norwegian Breast Cancer Screening Program. J Med Screen 23: 203-209.

10. Demoor-Goldschmidt C, Supiot S, Oberlin O, Helfre S, Vigneron C, et al. (2017) Clinical and diagnosis characteristics of breast cancers in women with a history of radiotherapy in the first 30years of life: A French multicentre cohort study. Radiother Oncol 124: 200-203.

11. Wallace WH, Blacklay A, Eiser C, Davies H, Hawkins M, et al. (2001) Developing strategies for long term follow up of survivors of childhood cancer. BMJ 323: 271-274.

12. Demoor-Goldschmidt C, Tabone MD, Bernier V, de Vathaire F, Berger C (2018) Long-term follow-up after childhood cancer in France supported by the SFCE-force and weakness-current state, results of a questionnaire and perspectives. $\mathrm{Br} J$ Radio 91: 20170819.

13. Berger C, El Fayech C, Pacquement H, Demoor-Goldschmidt C, Ducassou S, et al (2015) Objectives and organization for the long-term follow-up after childhood cancer. Bulletin du cancer 102: 579-585.

14. van de Poll-Franse LV, van Eenbergen MC (2008) Internet use by cancer survivors: current use and future wishes. Support Care Cancer 16: 1189-1195.

15. Oeffinger KC, Hudson MM, Mertens AC, Smith SM, Mitby PA, et al. (2011) Increasing rates of breast cancer and cardiac surveillance among high-risk survivors of childhood Hodgkin lymphoma following a mailed, one-page survivorship care plan. Pediatr Blood Cancer 56: 818-824.

16. Blaauwbroek R, Barf HA, Groenier KH, Kremer LC, van der Meer K, et al. (2012) Family doctor-driven follow-up for adult childhood cancer survivors supported by a web-based survivor care plan. J Cancer Surviv 6: 163-171.

17. Groen WG, Kuijpers W, Oldenburg HS, Wouters MW, Aaronson NK, et al. (2015) Empowerment of Cancer Survivors Through Information Technology: An Integrative Review. J Med Internet Res 17: e270. [Crossref]

18. Kuijpers W, Groen WG, Aaronson NK, van Harten WH (2013) A systematic review of web-based interventions for patient empowerment and physical activity in chronic diseases: relevance for cancer survivors. J Med Internet Res 15: e37. [Crossref]

19. Haupt R, Essiaf S, Dellacasa C, Ronckers CM, Caruso S, et al. (2018) The 'Survivorship Passport' for childhood cancer survivors. Eur J Cancer 102: 69-81.

20. Gramatges MM, Bonaduce de Nigris F, King J, Horowitz ME, et al. (2018) Improving Childhood Cancer Survivor Care Through Web-Based Platforms. Oncology 32: e1-e10. 
Demoor-Goldschmidt C (2020) Healthcare system barriers of breast and thyroid screening for childhood and young adulthood cancer survivors in France: A qualitative study of the DeNaCaPST programme at one year

21. Poplack DG, Fordis M, Landier W, Bhatia S, Hudson MM, et al. (2014) Childhood cancer survivor care: development of the Passport for Care. Nature reviews Clinical oncology 11: 740-750.

22. Horowitz ME, Fordis M, Krause S, McKellar J, Poplack DG (2009) Passport for care: implementing the survivorship care plan. J Oncol Pract 5: 110-112. [Crossref]
23. Song A, Fish JD (2018) Caring for survivors of childhood cancer: it takes a village. Curr Opin Pediatr 30: 864-873. [Crossref]

24. Volerman A (2015) Primary Care of the Childhood Cancer Survivor. Med Clin North Am 99: 1059-1073. [Crossref]

Copyright: (C2020 Demoor-Goldschmidt C. This is an open-access article distributed under the terms of the Creative Commons Attribution License, which permits unrestricted use, distribution, and reproduction in any medium, provided the original author and source are credited. 